\title{
SELF-DUALITY AND DIFFERENTIABLE STRUCTURES ON THE CONNECTED SUM OF COMPLEX PROJECTIVE PLANES
}

\author{
HENRIK PEDERSEN AND YAT SUN POON
}

(Communicated by Ronald Stern)

\begin{abstract}
It is proved that if the twistor space of a self-dual four-manifold of positive scalar curvature contains a real effective divisor of degree two, then the four-manifold is diffeomorphic to the connected sum $n \mathbb{C} P^{2}$ of $n$ complex projective planes for some $n$. It follows that if the four-manifold is known to be homeomorphic to $4 \mathbb{C} P^{2}$, then it is also diffeomorphic to $4 \mathbb{C} P^{2}$.
\end{abstract}

\section{INTRODUCTION.}

It was proved by Donaldson and Friedman [2] and independently by Floer [3] that on the connected sums $n \mathbb{C} P^{2}$ of $n$ complex projective planes there is a self-dual conformal class. Their existence theorem is nicely complemented by LeBrun's explicit construction [6]. However, LeBrun's conformal classes on $n \mathbb{C} P^{2}$ are so special that their associated twistor spaces have algebraic dimension three. In other words, the twistor spaces are Moishezon.

On the other hand, Campana showed [1] that if the twistor space $Z$ of a self-dual conformal class on a compact manifold $X$ is Moishezon, then $X$ is homeomorphic to $n \mathbb{C} P^{2}$ for some $n$. Therefore, the natural question is

Question 1. Is $X$ necessarily diffeomorphic to $n \mathbb{C} P^{2}$ ?

Note that when $Z$ is Moishezon, the conformal class of $X$ contains a metric of positive constant scalar curvature [9]. Hence we ask

Question 2. Suppose that $X$ is a smooth manifold homeomorphic to $n \mathbb{C} P^{2}$. If $X$ admits a self-dual metric of positive constant scalar curvature, is $X$ diffeomorphic to $n \mathbb{C} P^{2}$ ?

An affirmative answer to Question 2 will imply an affirmative answer to Question 1. Due to the work in [8] and [10], when $n=0,1,2$, and 3 , the answer to Question 2 is indeed affirmative. One way to prove this result is to find an effective elementary divisor in the twistor space. Then the restriction of the twistor fibration to this divisor will exhibit the differentiable structure on $X[10]$. By definition, an elementary divisor is a divisor whose intersection number with

Received by the editors October 19, 1992.

1991 Mathematics Subject Classification. Primary 32L25; Secondary 53A30, 53C15.

The second author was partially supported by the National Science Foundation grant no. DMS9103047. 
a real twistor line is equal to one. On a Moishezon space, there are plenty of effective divisors. Therefore, instead of considering elementary divisors, we may consider the case when there are real effective divisors whose intersection number with any real twistor line is equal to two. In the subsequent discussion, by the degree of a divisor in a twistor space, we mean the intersection number of the divisor with any twistor line.

Theorem 1. Suppose $X$ is a simply connected self-dual manifold of positive scalar curvature. If the twistor space $Z$ admits a real effective divisor of degree two, then $X$ is diffeomorphic to $n \mathbb{C} P^{2}$ for some $n$.

This theorem has the following immediate consequence.

Theorem 2. Suppose $X$ is homeomorphic to $4 \mathbb{C} P^{2}$. If $X$ is a self-dual manifold of positive scalar curvature, then $X$ is diffeomorphic to $4 \mathbb{C} P^{2}$.

Remark. Note that the geometric constraints on $X$ in Theorem 1 impose topological constraints on $X$, namely, the intersection form being positive definite. Due to Donaldson and Freedman's work, with the simply connectivity, $X$ is homeomorphic to $n \mathbb{C} P^{2}$.

\section{DEGREE TWO DIVISORS}

The twistor space $Z$ of a Riemannian four-manifold $X$ is the total space of the bundle of anti-self-dual 2-forms of unit length. The fibration $p$ from $Z$ onto $X$ is called the twistor fibration. The fibrewise antipodal map $\sigma$ is called the real structure. When the conformal class on $X$ is self-dual, $Z$ has a natural complex structure such that $\sigma$ is antiholomorphic and that the fibres of the twistor fibration are holomorphic rational curves in the complex manifold $Z$. These rational curves are called the real twistor lines. The normal bundle of any real twistor line is isomorphic to $\mathbf{H} \oplus \mathbf{H}$, where $\mathbf{H}$ is the degree-one line bundle on $\mathbb{C} P^{1}$.

On the twistor space $Z$, there is a naturally defined holomorphic line bundle $\mathbf{K}^{-1 / 2}$ on $Z$ such that $\left(\mathbf{K}^{-1 / 2}\right)^{2}=\mathbf{K}^{-1}$. We shall call $\mathbf{K}^{-1 / 2}$ the fundamental line bundle and the corresponding complete linear system the fundamental system. Since the fundamental line bundle is real, in the sense that the pull-back of $\mathbf{K}^{-1 / 2}$ by the real structure is conjugate linearly isomorphic to $\mathbf{K}^{-1 / 2}$, the fundamental system is real, and when it is nonempty, it contains real elements.

Lemma 2.1. Let $S$ be an effective degree-two divisor. If $S$ is real and irreducible, then it is nonsingular.

Proof. Due to the reality of $S$, it was proved in [5] that if $S$ is singular at one point $z$ in the twistor space, it is singular along the real twistor line $L$ through $z$. Also due to the reality and the assumption that $S$ is a degree-two divisor, there is a real line bundle $\mathbf{F}$ of vanishing real Chern class such that the associated line bundle of $S$ is $\mathbf{K}^{-1 / 2} \mathbf{F}$. Therefore, the section $S$ with $S$ as its zero divisor is an element of

$$
H^{0}\left(Z, \mathscr{I}^{2}\left(\mathbf{K}^{-1 / 2} \mathbf{F}\right)\right)
$$

where $\mathscr{I}$. is the ideal sheaf of the twistor line $L$ in the twistor space. Since the only section of $\mathbf{K}^{-1 / 2} \mathbf{F}$ vanishing along $L$ to order three is the zero section [5], the restriction map $r$ :

$$
r: H^{0}\left(Z, \mathscr{I}^{2}\left(\mathbf{K}^{-1 / 2} \mathbf{F}\right)\right) \rightarrow H^{0}\left(L, \mathscr{O}\left(\mathbf{K}^{-1 / 2} \mathbf{F} \otimes S^{2} \mathbf{N}^{*}\right)\right)
$$


is an injection, where $\mathbf{N}$ is the normal bundle of the twistor line $L$ in the twistor space.

By the construction of twistor spaces [5], it is known that

$$
\mathbf{N} \simeq \mathbf{H} \otimes\left(\mathbf{V}_{+}\right)_{x},
$$

where $\left(\mathbf{V}_{+}\right)_{x}$ is the fibre of the locally defined positive spinors on the manifold $X$ at the point $x=p(z)$. While the restriction of the fundamental bundle $\mathbf{K}^{-1 / 2}$ onto any twistor line is the bundle $\mathbf{H}^{2}$ and the bundle $\mathbf{F}$ is trivial on any real twistor line,

$$
H^{0}\left(L, \mathscr{O}\left(\mathbf{K}^{-1 / 2} \mathbf{F} \otimes S^{2} \mathbf{N}^{*}\right)\right)
$$

is naturally identified as the vector space $S^{2}\left(V_{+}^{*}\right)_{x}$. Therefore, the section $s$ is uniquely determined by $r(s)$ as a quadratic polynomial on $\left(V_{+}\right)_{x}$. Then the divisor $S$ is uniquely determined by the zeros of this quadratic polynomial $r(s)$. Since $S$ is real, the zeros of $r(s)$ form a conjugate pair.

On the other hand, in order to resolve the singularity of $S$ along $L$, we blow up the twistor space $Z$ along $L$. Then the exceptional divisor of the blowing up is the projectivized normal bundle over $L$, so it is a quadric $Q$ naturally identified with $L \times P\left(\left(V_{+}\right)_{x}\right)$. Since $L$ is the projectivized negative spinors over $x$, i.e., $P\left(\left(V_{-}\right)_{x}\right)$, the real structure on $Z$ extends over the blow-up space $\hat{Z}$, so the induced real structure on

$$
L \times P\left(\left(V_{+}\right)_{x}\right) \simeq P\left(\left(V_{-}\right)_{x}\right) \times P\left(\left(V_{+}\right)_{x}\right)
$$

is the antipodal map on each factor of this product. Since $s$ vanishes along $L$ to order two, the proper transform $\hat{S}$ of $S$ will intersect the exceptional quadric $Q$. As $S$ is uniquely determined by a conjugate pair $u$ and $\bar{u}$ of zeros of $r(s)$ on $\left(V_{+}\right)_{x}, \bar{S}$ intersects $Q$ along a conjugate pair of curves, namely

$$
C:=P\left(\left(V_{-}\right)_{x} \times u \text { and } \bar{C}:=P\left(\left(V_{-}\right)_{x}\right) \times \bar{u} .\right.
$$

In particular, the singularity of $S$ along $L$ is completely resolved by blowing up $L$. Since real twistor lines are mutually disjoint and $S$ can only be singular along finitely many real twistor lines, after blowing up finitely many real twistor lines on the twistor space, the proper transform of $S$ to $\hat{S}$ is a nonsingular irreducible surface.

To calculate the self-intersection number of $C$ on the smooth surface $\hat{S}$, we may assume that $S$ contains only one twistor line as its singularity. In this case, $\hat{S}$ is a member of the system $\left|-\frac{1}{2} K+F-2 Q\right|$. By the adjunction formula,

$$
-K_{\hat{S}}=-K_{\hat{Z}}-\hat{S}=-K-Q+\frac{1}{2} K-F+2 Q=-\frac{1}{2} K-F+Q .
$$

Therefore, the self-intersection number on $\hat{S}$ is

$$
\begin{aligned}
C^{2} & =-2-K_{\hat{S}} \cdot C=-2-\frac{1}{2} K \cdot C-F \cdot C+Q \cdot C \\
& =-2-\frac{1}{2} K \cdot L-F \cdot L+Q \cdot C=-2+2-0+1=1 .
\end{aligned}
$$

Since $C$ is a curve with positive self-intersection number on $\hat{S}, \hat{S}$ is necessarily a projective algebraic surface. On the other hand, by reality, $\bar{C}$ is a curve on $\hat{S}$, which has all the features of $C$. In particular, $\bar{C}^{2}=1$. Yet, since $C$ and $\bar{C}$ are two generators of the same family on the exceptional quadric $Q$, they are disjoint on $\hat{S}$. This is a contradiction to the Hodge index theorem stating that 
irreducible curves with positive self-intersection on projective algebraic surfaces must intersect. Therefore, $S$ is nonsingular.

\section{PROOF OF THE THEOREMS}

To prove Theorem 1, we assume there is a real degree two effective divisor $S$ in $Z$. Due to Lemma 2.1 , if $S$ is singular, it is reducible to the sum of a conjugate pair of effective elementary divisors $D$ and $\bar{D}$ [9]. By [Lemma 1.10, 9], $D$ contains a unique real twistor line $L$ such that the associated map of the complete liner system $|L|$ on $D$ exhibits $D$ as a blow up of $\mathbb{C} P^{2} n$ times such that $L$ is linearly equivalent to the proper transform of the hyperplane class on $\mathbb{C} P^{2}$. Let $W$ be a differentiable chart on $X$ with $p(L)$ as center and $U=p^{-1}(W)$. Then $U$ intersects $D$ along a tubular neighborhood of $L$ in $D$. We can choose $W$ so small that the intersection $U \cap D$ does not meet any exceptional divisors of the blow up from $\mathbb{C} P^{2}$ to $D$. Then $D \backslash U$ is diffeomorphic to $\mathbf{R}^{4} \# n \mathbb{C} P^{2}$. Since the associated bundle of $D$ has degree one on any real twistor line, $D$ intersects any real twistor line transversely at one point except when the twistor line is the unique $L$ contained in $D$. Therefore, the twistor fibration $p$ is a diffeomorphism from $D \backslash U$ to $X \backslash W$. Hence, $X$ is diffeomorphic to $S^{4} \# n \mathbb{C} P^{2}$, which is $n \mathbb{C} P^{2}$. This is a result already observed in [7] and [10].

What we need to study next is the case when all the real degree-two effective divisors are irreducible. By Lemma 2.1, they are actually nonsingular. Given the assumptions on $X$, it is not hard to repeat the arguments in [5] to show that when $S$ is a nonsingular irreducible element of the fundamental system, $S$ is the blow-up of a rational ruled surface $2 n$ times, such that $S$ contains a real twistor line as an irreducible fibre of the ruling.

To describe the structure of $S$ in details we recall that $S$ is real, and hence the anticanonical bundle $\mathbf{K}_{S}^{-1}$ of $S$ is real. Since the intersection number on $S$ of $-K_{S}$ with any exceptional divisor of the blowing up is equal to one, none of the exceptional divisors is real. If $E$ is the last exceptional divisor of the blowing up, it is an irreducible $(-1)$-curve. Its conjugate $\bar{E}$ is also an irreducible (-1)-curve. As $S$ is a blow-up of a rational surface, $E$ can be realized as an irreducible component of a reducible fibre of the ruling. By the reality of the fibre which is also a real twistor line, $\bar{E}$ is also a component of a reducible fibre. Since different components of reducible fibres can intersect at most one point and the intersection number of $E$ and $\bar{E}$ is even, $E$ is disjoint from $\bar{E}$. Therefore, this pair of $(-1)$-curves can be blown down simultaneously. The resulting surface inherits a real structure without real points. This process of blowing down a conjugate pair of mutually disjoint exceptional divisors can be carried on until $S$ is blown down to a real minimal surface $Q$.

By construction, $Q$ is a ruled surface; its infinity section is the unique rational curve with negative self-intersection. Since the infinity section intersects a generic fibre once, it is not real. Therefore, the existence of a real structure on $Q$ implies that $Q$ is a quadric surface. To recognize the real structure on $Q$, recall that there is a real twistor line $L$ on $S$ as an irreducible fibre of the ruling. Therefore, $Q$ is naturally isomorphic to

$$
C \times L:=\mathbb{P}\left(H^{0}(S, \mathscr{O}(L))^{*}\right) \times L .
$$


The real structure on $H^{0}(S, \mathscr{O}(L))$ is the complex conjugation. The real structure on $C \times L$ is the complex conjugation on $C$ and the antipodal map on $L$.

The above description shows clearly that there is an $S^{1}$-family of real twistor lines on the surface $S$. They are parametrized by an equator of the Riemann sphere $C$. Removing all these real twistor lines on $S$, we have an open submanifold $S_{0}$ as an unbranched double covering of $X$ with a copy of an embedded $S^{1}$ removed. This is an unbranched double covering because, by the reality of $S$, when a real twistor line is not contained in the complex surface $S$, it intersects $S$ transversely at a conjugate pair of points.

On the other hand, as $S$ is the blow-up of $C \times L 2 n$ times and real twistor lines do not pass through any points of blowing up, $S_{0}$ is diffeomorphic to the disjoint union

$$
\left[\left(D^{+} \times S^{2}\right) \coprod\left(D^{-} \times S^{2}\right)\right] \# 2 n \mathbb{C} P^{2},
$$

where $D^{+}$and $D^{-}$are the upper and lower hemispheres of the Riemann sphere $C$ with respect to the equator which parametrizes real twistor lines in $S$. As $S_{0}$ is a double covering of $X \backslash S^{1}$, this is possible only when one deck of the covering is $\left(D^{+} \times S^{2}\right) \# n \mathbb{C} P^{2}$ and the other deck is $\left(D^{-} \times S^{2}\right) \# n \mathbb{C} P^{2}$, i.e., $X \backslash S^{1}$ is diffeomorphic, via the twistor fibration, to $\left(D^{+} \times S^{2}\right) \# n \mathbb{C} P^{2}$ and $\left(D^{-} \times S^{2}\right) \# n \mathbb{C} P^{2}$.

On the other hand, when $S$ is blown down to $\mathbb{C} P^{1} \times \mathbb{C} P^{1}, S_{0}$ is blown down to $\left(D^{+} \times S^{2}\right) \amalg\left(D^{-} \times S^{2}\right)$. This is a double covering of $S^{4}$ with a circle removed. The blow-down of $S$ is identical to $\mathbb{C} P^{1} \times \mathbb{C} P^{1}$ as a real fundamental divisor of the twistor space $\mathbb{C} P^{3}$, and the restriction of the twistor fibration to $\mathbb{C} P^{1} \times \mathbb{C} P^{1}$ gives a smooth compactification of a deck, say $D^{+} \times S^{2}$, by a circle to $S^{4}$. Thus $X$ is smoothly compactified by a circle to $S^{4} \# n \mathbb{C} P^{2}$, i.e., $n \mathbb{C} P^{2}$. Our proof of Theorem 1 is complete.

Finally, when $X$ is homeomorphic to $4 \mathbb{C} P^{2}$, the topological data on $Z$, such as the Chern numbers, are available [5]

$$
c_{1}^{3}=0, \quad c_{1} c_{2}=24, \quad c_{3}=12 .
$$

By the Riemann-Roch formula,

$$
\chi\left(Z, \mathbf{K}^{-1 / 2}\right)=2 .
$$

Since $\mathbf{K} \otimes K^{1 / 2}$ is a bundle of negative degree on any real twistor line, its only section over the twistor space is zero. By Serre duality, on any twistor space, $h^{3}\left(Z, \mathbf{K}^{-1 / 2}\right)=0$. When the self-dual conformal class contains a metric of positive scalar curvature, Hitchin's vanishing theorem [4], together with Serre duality, implies that $h^{2}\left(Z, \mathbf{K}^{-1 / 2}\right)=0$. Therefore,

$$
h^{0}\left(Z, \mathbf{K}^{-1 / 2}\right)-h^{1}\left(Z, \mathbf{K}^{-1 / 2}\right)=2 .
$$

In particular, the fundamental system $\left|-\frac{1}{2} K\right|$ is at least as big as a pencil. Therefore, Theorem 2 is a direct consequence of Theorem 1 and the existence of effective degree two divisors.

\section{ACKNOWLEDGMENT}

The first author thanks the Department of Mathematics at Riverside for hospitality. 


\section{REFERENCES}

1. F. Campana, On twistor spaces of the class $\mathscr{C}$, J. Differential Geom. 33 (1991), 541-549.

2. S. K. Donaldson and R. Friedman, Connected sums of self-dual manifolds and deformations of singular spaces, Nonlinearity 2 (1989), 197-239.

3. A. Floer, Self-dual conformal structure on $\ell C P^{2}$, J. Differential Geom. 33 (1991), 551-574.

4. N. J. Hitchin, Linear field equations on self-dual spaces, Proc. London Math. Soc. (3) A370 (1980), $173-191$.

5. __ Kählerian twistor spaces, Proc. London Math. Soc. (3) 3 (1981), 133-150.

6. C. LeBrun, Explicit self-dual metrics on $C P_{2} \# \cdots \# C P_{2}$, J. Differential Geom. 34 (1991), 223-254.

7. C. LeBrun and Y. S. Poon, Twistors, Kähler manifolds and bimeromorphic geometry. II, J. Amer. Math. Soc. 5 (1992), 317-325.

8. Y. S. Poon, Compact self-dual manifolds with positive scaler curvature, J. Differential Geom. 24 (1986), 97-132.

9. __ On the algebraic dimension of twistor spaces, Math. Ann. 282 (1988), 621- 627.

10. _ On the algebraic structure of twistor spaces, J. Differential Geom. 36 (1992), 451-491.

Department of Mathematics and Computer Science, Odense University, 5230 Odense M, DenMarK

E-mail address: henrik@imada.ou.dk

Department of Mathematics, University of California, Riverside, California 92521

E-mail address: ypoon@math.ucr.edu 